\title{
Tecnologías emergentes para el desarrollo de competencias ciudadanas en estudiantes de licenciatura
}

\section{Emerging technologias for the development of citizenship skills in undergraduate students}

\author{
Flavia Alicia Juárez Manzano \\ Marco Antonio Velázquez Albo² \\ BUAP, México
}

\section{RESUMEN}

El uso de la tecnología en los últimos años se ha masificado en la sociedad. En el caso de la poblaciónescolarizadautilizan latecnologíacomo herramientas que les facilitan el aprendizaje. En este artículo se exploraron las opiniones de los estudiantes de licenciatura de una universidad pública de México, respecto del uso de la

1 Docente en la Facultad de Lenguas BUAP, Maestría en Docencia Universitaria por la Universidad Iberoamericana Puebla, Estudiante del Doctorado en Investigación e Innovación Educativa por la BUAP, Docente de Tronco Común BUAP. ORCID https://orcid.org/0000-0001-72614122 ali07 juarez@yahoo.com.mx

2 Profesor Tiempo Completo en el Doctorado en Investigación e Innovación Educativa de la Benemérita Universidad Autónoma de Puebla, Puebla. México ORCID http://orcid.org/0000-0002-5916-4283/print marcovelazquez_buap@yahoo.com.mx plataforma Schoology como herramienta para el aprendizaje de competencias ciudadanas, durante la crisis covid-19 a nivel mundial. La metodología utilizada fue cualitativa, el estudio se realizó a 51 participantes, se dio seguimiento a las actividades que realizaron durante un curso denominado Formación Humana y Social, se aplicó un cuestionario para conocer su opinión mediante el formulario de Google. Los resultados que se obtuvieron permitieron observar que los participantes no hicieron uso de la plataforma Schoology para la realización de actividades de la materia, aunque ellos opinaron que es una plataforma que les permite aprender los contenidos de la materia. Como conclusión se pudo observar que la tecnología puede 
ayudar ante situaciones de crisis, sin embargo, el cambio de un sistema escolar presencial a uno a distancia mediado por tecnología no puede garantizar el desarrollo de competencias ciudadanas.

PALABRAS CLAVE: Plataformas educativas, Competencias, Ciudadanía, Ciberespacio, Tecnología educativa.

\section{SUMMARY}

The use of technology in recent years has become widespread in society, in the case of the school population use technology as tools to facilitate learning. In this article we explored the opinions of undergraduate students from a public university in Mexico, regarding the use of the Schoology platform as a tool for learning citizenship skills, during the covid-19 crisis worldwide. The methodology used was qualitative, the study was carried out on 51 participants, the activities they carried out during a course called Human and Social Training were followed up, a questionnaire was applied to find out their opinion using the Google form. The results obtained allowed us to observe that the participants did not use the Schoology platform to carry out activities on the subject, although they thought that it was a platform that allowed them to learn the contents of the subject. As a conclusion, it could be observed that technology can help in crisis situations, however, the change from a face-to-face school system to a distance one mediated by technology cannot guarantee the development of citizen competencias.

KEIWORDS: Educational platforms, Competencies, Citizenship, Cyberspace, Educational technology.

\section{INTRODUCCIÓN}

El uso de herramientas tecnológicas como computadora, celular, tablet, internet, etc., se han masificado en los últimos tiempos, en la Educación Superior algunas de estas herramientas se han hecho indispensables para cursar estos estudios. De acuerdo al Instituto Nacional de Estadística y Geografía (INEGI, 2019) y de acuerdo a las estadísticas aplicadas a diversos sectores de la población se encontró que el $95.1 \%$ de la población con estudios de nivel superior (licenciatura o posgrado) usa internet, el $44.9 \%$ de la población cuenta con computadora en el hogar, el $52.9 \%$ puede acceder a internet.

Uno de los objetivos de la universidad es la producción del conocimiento científico, por lo que la tecnología se ha convertido en un recurso muy importante para la generación del mismo. La escuela y las formas de aprender han tenido que adaptarse a los nuevos tiempos y agregar la tecnología como parte de los recursos para promover el aprendizaje en los estudiantes.

La incorporación de la tecnología en la educación en los últimos años se ha incrementado. Se tiene la creencia de que los alumnos son más hábiles en el uso de la tecnología por estar en contacto con ella de forma permanente, a diferencia de los profesores quienes en ocasiones les cuesta trabajo adaptarse e incorporar la tecnología a sus actividades docentes.

Ante estos planteamientos, se ha propuesto como objetivo conocer las opiniones de los estudiantes de licenciatura de una universidad pública de México, respecto del uso de la plataforma Schoology como herramienta para el aprendizaje de competencias ciudadanas, durante la crisis covid- 19 .

\section{TECNOLOGÍAS EN EDUCACIÓN}

La computadora ha evolucionado de manera acelerada, así, por ejemplo, que un tiempo fueron grandes aparatos operados mediante implementos complejos para su aplicación y que era imposible su traslado o movimiento, en comparación con las computadoras que se usan 
actualmente, las cuales son fáciles de operar y trasladar.

El internet que en sus primeros tiempos se utilizó por medio de conexión telefónica, en comparación con la señal de internet que se usa en la actualidad es rápido, inalámbrico, y con una velocidad inimaginable para esos tiempos.

La educación mediada por tecnología en México fue la que se impartió en el sistema escolar denominado Enseñanza Secundaria por Televisión y posteriormente denominada Telesecundaria, sus inicios datan de mediados de los años 60 . El objetivo de este sistema de educación fue la erradicación del atraso que tenían las comunidades rurales, indígenas y zonas urbano marginadas de México y que por sus características geográficas y/o económicas era imposible el establecimiento de sistemas educativos de otras modalidades.

La característica de este sistema de Telesecundaria era que se hacía uso de programas grabados, los maestros no tenían una formación profesional específica con este modelo educativo, se optó por la capacitación de profesores de nuevo ingreso y la actualización de los que ya atendían esta modalidad haciendo uso de los mismos recursos que usaban para los alumnos.

Las clases se impartían en salones conocidos como teleaulas y los telemaestros quienes explicaban los contenidos a través de capsulas programadas que duraban aproximadamente 15 minutos y un coordinador que se encontraba dentro de la teleaula un encargado de reforzar y orientar a los estudiantes sobre las actividades que se solicitaban. Secretaría de Educación Pública (SEP. 2011, p. 2).

Los cambios económicos que ocurrieron en la última década del siglo veinte a nivel mundial, han impactado en diversos ámbitos de la sociedad. Algunos organismos internacionales como la Organización para la Cooperación y el Desarrollo Económico (OCDE), la Organización de las Naciones Unidas para la Educación la Ciencia y la Cultura (UNESCO), han centrado su interés en recomendar ciertas políticas que permitan corregir algunas las fallas que presentan los sistemas educativos mediante el uso de la tecnología para subsanar dichas fallas. (Reyes, 2012, p. 15).

En los últimos años México ha logrado grandes avances en materia educativa, muchas instituciones de educación superior se han consolidaron mediante la formación de centros de investigación y formación de profesionistas que han podido impactar en diversos ámbitos de la sociedad y contribuir con el desarrollo del país con el uso de la tecnología y el acceso a la información para la generación de conocimiento.

El Plan Sectorial de Educación (2007-2012) mostró los avances que él país había logrado en las últimas décadas en términos de educación y los grandes rezagos en esta misma materia que prevalecían y que se debían atender para lograr avanzar en el desarrollo del país. Ante esta necesidad se propuso la incorporación de las tecnologías de la información y la comunicación como recurso didáctico que permitiera la integración de sus ciudadanos a la sociedad del conocimiento. (SEP, 2007).

Tras la incorporación de la tecnología al ámbito académico, muchos de los contenidos curriculares se han automatizado, Los actores de la educación como maestros y autoridades han comprendido que la generación del conocimiento no sólo ocurre en el aula, a través del docente o a través de un libro físico que se encuentra en una biblioteca, mucha información ha sido colocada en el ciberespacio, para ser consultada y usada por quien la requiere, lo que ha revolucionado las formas de acceder al conocimiento y las formas de aprender de los estudiantes. 
El uso de las tecnologías que se incorporan al ámbito de la educación, no ha impactado exclusivamente en las formas de aprender de los alumnos, sino en las nuevas formas de relacionarse e interactuar entre ellos mismos y con otros actores de la educción, así también han impactado en la forma en que se adquiere la información o la manera en que se puede comprobar que el alumno se apropia del conocimiento y adquiere las competencias de su profesión.

\section{CIBERESPACIO Y TECNOLOGÍA EMERGENTE}

Con la evolución de la tecnología se ha llegado ahora al concepto de nuevas tecnologías o tecnologías emergentes. Concari (2014) define tecnología emergente como aquellas que se refiere al uso de nuevas tecnologías con potencial de demostrarse como tecnologías disruptivas. Construye innovaciones en desarrollo que en un futuro cambiarían la forma de vivir y de producir brindando mayor facilidad a la hora de realizar tareas o haciéndolas más seguras. (p.495).

El ciberespacio considerado como uno de los elementos de la tecnología y fundamental para el logro de los objetivos educativos en la actualidad, es definido por la Organización de Estados Iberoamericanos para la Educación, la Ciencia y la Cultura (OEI) (2015) como:

un espacio virtual de interacción, existe solamente como espacio relacional; su realidad se construye a través del intercambio de información; es decir, es espacio y es medio. Una red sin interacción entre sus miembros deja de ser una red; la red existe porque existen relaciones entre sus integrantes. Ciertas tareas que antes era necesario realizar físicamente, ahora se pueden realizar a través de escenarios virtuales alojados en el Ciberespacio. En muchos casos, la eficacia del mundo virtual ha hecho desapareceré del mundo real elementos que no hace mucho parecían firmemente anclados en nuestro entorno material (pár. 1).

El escenario tradicional de aprendizaje se ha transformado, de ser un espacio físico donde se encuentra el maestro, los alumnos y donde se gestionan los aprendizajes, ahora el ciberespacio considerado como el nuevo ámbito para albergar información o materiales que pueden ser gestionados por el maestro o por los propios alumnos y así propiciar el aprendizaje.

El mundo actual exige que las personas sean autogestivas de su propio aprendizaje y para el logro de tal objetivo, debe no basta con allegarse de información que se encuentra en el ciberespacio, sino que es necesario que tengan claro lo que buscan, saber cómo organizar y manejar los datos que se encuentran albergados en sitios de internet, así ante las nuevas situaciones que surgen en el mundo, movimientos sociales, fenómenos naturales, nuevas enfermedades que hacen que no se pueda continuar con los estudios que se imparten de forma tradicional.

Estas situaciones hacen que las instituciones educativas busquen de manera rápida $\mathrm{O}$ alternativa como alguna solución como puede ser la educación a distancia o virtual, la tecnología emergente como plataformas educativas de acceso libre o no como Schoology apoyen a resolver cierto tipo de necesidades educativas.

\section{SOCIEDAD DEL CONOCIMIENTO Y TECNOLOGÍAS EMERGENTES}

La sociedad de la información y sociedad del conocimiento son conceptos que surgieron con la tecnología y se refieren al uso de "dispositivos digitales para facilitar el aprendizaje y consolidar un modelo integral de educación que cumpla con los objetivos tecno-pedagógicos de la actualidad" (Pérez, Mercado, Martínez, y Mena 2018, p 48). 
Las competencias relacionadas con el uso de tecnología son consideradas como competencias fundamentales en la sociedad actual. Las plataformas educativas como Schoology son consideradas como tecnologías emergentes, por sus características funcionales y pueden ser utilizadas como recursos de aprendizaje, que facilitan la organización de información que debe ponerse a disposición de los aprendices y que les ayudarán en su desarrollo de competencias genéricas y profesionales.

Las competencias clave del Proyecto DeSeCo señala que "los individuos deben poder usar un amplio rango de herramientas para interactuar efectivamente con el ambiente: tanto físico como en la tecnología de la información y socioculturales como en el uso del lenguaje" (p. 4), así para el logro de los propósitos de aprendizaje, es necesario que los estudiantes desarrollen diversas competencias entre las que están relacionadas con el uso de tecnología de la información y la comunicación.

Por las características propias que tiene la tecnología, la cual se encuentra en una constante actualización y surgimiento de nuevas herramientas que cada día facilitan la vida de las personas, hace que los estudiantes no solo adquieran conocimientos sobre el manejo de tecnología específica o que se usa en ese momento, sino que es necesario que esté se mentalice de la importancia de estar abierto a adquirir nuevos conocimientos y adaptarse a los nuevos requerimientos que marca la sociedad.

Para Valencia, Serna, Ochoa, Caicedo, Montes, y Chávez, (2016) afirman que:

Las competencias en el diseño de escenarios educativos apoyados en TIC se refieren a las habilidades de planificación y organización de elementos que permitan la construcción de escenarios educativos apoyados en TIC para el aprendizaje significativo y la formación integral del estudiante (p.17).

Se puede decir que actualmente es fácil encontrar información en la red casi de todo tipo, en muchos casos basta escribir en un buscador como Google para tener a la mano un gran número de información relacionada con el tema que se desea consultar, cantidades que en un momento dado sería imposible para el interesado poder consultar y emitir un juicio después de discriminar lo que puede ser útil o no de acuerdo a sus intereses.

Ante esto es necesario que los interesados en buscar información no solo sean competentes en el manejo del equipo de cómputo sino además necesita contar con otras competencias que le permitan discriminar la información que requieren en un tiempo razonable y poder hacer uso de ella conocido como Alfabetismo informacional

\section{APRENDIZAJE DE COMPETENCIAS CIUDADANAS}

El surgimiento de la tecnología a nivel mundial, ha hecho que las formas en que se aprende hayan cambiado, actualmente el aprendizaje no se desarrolla exclusivamente en un aula, el internet se ha convertido en uno de los espacios donde se puede obtener información de diversos temas, actualmente si una persona desea aprender un tema basta con buscar algún material, sea video o texto para informarse y aprender lo que se desea.

La escuela presencial desarrollaba competencias ciudadanas desde el momento en que interactuaban maestros, alumnos y autoridades educativas y así, cada día se desarrollaban competencias ciudadanas las cuales se ejercían y adquirían por la convivencia frecuente. Las clases virtuales o a distancia no permiten una interacción física entre personas, sin embargo, con el uso de la tecnología se hace necesario 
considerar y ejercer ciertas normas y reglas que permitan el buen desempeño de cursos aunque sean virtuales o mediados por tecnología.

Ante esto la escuela y el profesor han tenido que adaptarse a las nuevas formas de aprender surgiendo nuevas formas de convivencia que están relacionadas con la ciudadanía o competencias ciudadanas que debe aprender quien hace uso de la tecnología. Autores como García y González (2014) plantean que las competencias ciudadanas "son un conjunto de conocimientos y habilidades de diversa índole que articuladas permiten la participación de los sujetos en las sociedades democráticas". ( $p$. 373).

Al hablar del desarrollo de competencias ciudadanas, el uso de plataformas educativas propicia que los alumnos al participar en una plataforma educativa tienen una participación activa ya que debido a que por las características que tienen las plataformas educativas hace uso de sus competencias ciudadanas como la competencia comunicativa que al usar la tecnología la comunicación es fundamental para la realización de actividades que se proponen en este tipo de espacios de aprendizaje.

Para Zambrano, Fernández, Rivera, y Zapata (2014) las competencias ciudadanas son

aquellos conocimientos y capacidades, que el individuo debe desarrollar para que le permita tomar conciencia individual y colectiva sobre sus actitudes y acciones realizadas en un contexto determinado, teniendo como referencias la ley y sus principios morales y religiosos, para conseguir la realización de su ser, en el instante que escucha, se comunica e interactúa con otros y respeta la naturaleza. (p.80).
Algunos autores como Caballero (2016) consideran que las competencias ciudadanas no son competencias que se construyan en un solo momento, sino que implican una formación permanente que requiere de otras competencias como la reflexión que hace el sujeto y que construye a lo largo de su vida, encaminado hacia la construcción de una ciudadanía Global, Alcántara (2017), producto del rompimiento de fronteras que han hecho las tecnologías emergentes.

\section{METODOLOGÍA}

La presente investigación fue un estudio cualitativo que se realizó con dos grupos de estudiantes de segundo semestre de Licenciatura en Enseñanza del Inglés, de la Facultad de Lenguas de la Benemérita Universidad Autónoma de Puebla, uno de los grupos tenía 28 estudiantes y en el otro grupo 23 estudiantes, en total se tomó a 51 participantes; el rango de edad de los participantes osciló entre los 18 a los 26 años de edad.

El instrumento se diseñó y puso a disposición de los participantes con la herramienta Google Forms, las actividades del curso Formación Humana y Social se subieron a la plataforma Schoology, se pidió a los participantes que ingresaran al enlace y respondieran el cuestionario, posteriormente se categorizaron las preguntas en tres grupos: datos generales de los participantes, uso de la plataforma Schoology y competencias ciudadanas. Se concentraron las tareas y actividades que debieron entregar los participantes durante el curso de la materia, posteriormente se analizaron las entregas.

Los participantes fueron alumnos que estaban inscritos en la materia Formación Humana y Social. Desde el inicio del curso los participantes utilizaban la plataforma Schoology para la entrega de tareas, las actividades de la materia en los dos primeros meses se realizaron de manera presencial, y la plataforma solo era 
usada para subir tareas, lecturas, materiales, etc., y las actividades de reflexión o análisis de materiales eran realizados en las sesiones presenciales.

Posteriormente al inicio de la pandemia Covid-19 y la suspensión de actividades a nivel nacional, se continuó con el curso de manera virtual y se sumaron otras actividades como videos reflexiones, foros a la plataforma para cubrir los objetivos de la materia.

Se monitoreó la plataforma Schoology, como lo muestra la imagen 1 y 2, durante el tiempo que duró el curso, para conocer cómo los participantes hacían uso de esta plataforma para la realización de actividades que se propusieron en el curso. Se aplicó al final del curso un cuestionario de 12 preguntas abiertas para conocer la opinión de los participantes sobre su experiencia al usar la plataforma. Durante el curso se apoyó con el uso de WhatsApp para resolver dudas sobre actividades de la plataforma.

Los participantes fueron estudiantes de la Licenciatura en Enseñanza del Inglés de la Facultad de Lenguas de la Benemérita Universidad Autónoma de Puebla, esta facultad imparte dos licenciaturas del sistema escolarizado: Licenciatura en Enseñanza del Inglés y Licenciatura en Enseñanza del Frances El plan de estudios de estas licenciaturas, señalan como parte de las materias curriculares la Formación Humana y Social que deben cursar los alumnos en el segundo semestre de la carrera y que en los siguientes semestres se desarrollan como ejes transversales.

El Modelo Universitario Minerva, (BUAP) en el programa de la materia Formación Humana y Social declara que los alumnos para desarrollar la competencia genérica del este eje transversal

Participa de manera comprometida dentro de su medio sociocultural para contribuir al desarrollo social, la preservación del medio ambiente y el cuidado de la salud, considerando los lenguajes científicos, tecnológicos y artísticos de su disciplina profesional al colaborar en la solución de problemas de manera interdisciplinaria (2016, p. 2).

La elección de la plataforma Schoology como recurso para impartir la materia fue por las herramientas con que cuenta, por ser una plataforma gratuita, otra característica es por la facilidad que permite de organizar por carpetas los temas, el poder adjuntar documentos, videos, enlaces, la posibilidad de llevar un control de asistencia, fechas de entrega de tareas, lista de tareas y calificaciones y por ser una plataforma de libre acceso

Antes de elegir la plataforma Schoology se exploraron otras plataformas como Classroom y Edmodo pero se optó por la plataforma Schoology por las características antes señaladas. Desde el inicio del curso se les indicó a los participantes que se trabajaría con esta plataforma y que en ella se subirían trabajos, lecturas y tareas, por la pandemia covid-19 se continuó con el curso y se retiraron las clases presenciales.

Se elaboró un concentrado de actividades y tareas en Excel para conocer las entregas de cada participante y contrastar con sus opiniones del cuestionario que se les aplicó.

\section{RESULTADOS}

Del total de alumnos inscritos al curso Formación Humana y Social, 40 participantes resolvieron el cuestionario, de los que el $80 \%$ fueron participantes del género femenino y $20 \%$ correspondió al género masculino. el $92.5 \%$ de los participantes consideraron que la plataforma es fácil y el $7.5 \%$ opinó que es difícil trabajar en ella. Al inicio los alumnos se les dificultó el uso de la herramienta por lo que algunas tareas las colocaban en otros espacios como el foro o en 
el espacio de comentarios, lo que dificultó el registro de entregas.

Los participantes que opinaron que es fácil el uso de la plataforma Schoology fue porque no tuvieron ningún problema en su uso, mientras que los que no les pareció fácil argumentaron que se debe a que la plataforma no emite mensajes para notificar cuando hay tareas o actividades nuevas, lo que hace que el participante deba estar al pendiente de la plataforma y en algunos casos si se descuidaba podía perder la entrega de tareas.

Una de las dificultades que encontraron es que aun cuando puede bajarse la aplicación de Schoology al teléfono móvil es difícil acceder desde esta herramienta, a veces era difícil cargar las actividades o documentos o hay quien argumentó que le pareció confusa la plataforma.

Los participantes opinaron que es una plataforma fácil en su uso y solo un participante opinó que le pareció aburrida la plataforma, dentro de los que opinaron que es fácil un participante argumentó lo siguiente: creo que es una página fácil de usar para subir trabajos y tareas, pero es mejor que las clases sean presenciales.

De las aplicaciones y herramientas con que cuenta la plataforma Schoology los participantes consideraron que es adecuado poder acceder a videos, cuestionarios, foro, enlaces y actividades, lo que enunciaron correspondió a las aplicaciones que se utilizaron, aunque la plataforma tiene otras aplicaciones los participantes no las mencionaron por lo que se aprecia que no exploraron la plataforma y sólo utilizaron las que se les propusieron.

Respecto a esta misma pregunta una opinión de los participantes fue: "Aunque el foro es bueno también puede ser malo ya que implica que otros compañeros pueden robar las ideas de aquellos que subieron las actividades primero $y$ por lo tanto no aprenden sobre el tema que se esté viendo". Esto nos permitió observar que el uso de herramientas como el foro permite a los participantes reflexionar sobre las prácticas éticas o antiéticas que llevan a cabo sus compañeros desarrollando así competencias ciudadanas.

En la pregunta de los contenidos y actividades propuestas en el curso cuál es la que te ayuda a ser mejor ciudadano los participantes opinaron que fue el tema de identidad, reflexiones para la vida, reconocimiento de emociones, las lecturas que se propusieron le parecieron útiles para su vida, todo lo que se planteó en el curso fue adecuado, sobre la otredad y la autoeficacia, la inclusión social, relaciones efectivas, regreso a la nueva normalidad y la integración social.

Los participantes consideraron que no todas las actividades que se realizaron como parte de los contenidos de la materia les ayudaron a desarrollar competencias ciudadanas como en el caso de la pregunta: Los participantes opinaron que fue el de instrumentos musicales, porque fueron actividades muy simples, como las siguientes: la actividad de "el día en que naci" se le hizo incomoda, la caja musical, porque no le encontró utilidad, la actividad de los frijoles, las tablas y los reportes de lectura porque considera que una lectura no le ayuda a ser mejor ciudadano.

Otra de las actividades que consideraron que no contribuyeron a la formación ciudadana fue la de Covid-19, uno de los participantes opinó que: Esas (actividades) no me hacian sentir de alguna manera especial porque es algo que me parece muy irrelevante para ser un mejor ciudadano, solamente hay que seguir las normas de salud y así cuidamos a otros y a uno mismo'.

Respecto de cómo ayuda la plataforma Schoology para el fomento de buenas prácticas ciudadanas, los participantes consideraron que les ayudó mucho porque les permitió el contacto con otros participantes, les enriquece 
el conocimiento, el respeto a otras opiniones, les enseñó a ser humanos, comparar ideas y respetar, les permitió reflexionar, aprender otros temas, les permitió opinar, mantenerse informados, tener contacto con alumnos y profesores, fue útil de para esta situación que vivimos actualmente pero que se debe pensar en los que no tienen internet.

También consideraron que les ayuda a no exponerse ante la pandemia que se vive y que pueden trabajar desde casa y una persona que opinó que no es la plataforma lo que les ayuda sino las actividades que se colocan en ella.

El $72.5 \%$ de los participantes recomendarían la plataforma para aprender sobre la Formación Humana y Social y el $27.5 \%$ dijo que tal vez. Por lo que se puede decir que es considerada como una buena herramienta tecnológica.

Al analizar la entrega de tareas y actividades se observa que las entregas fueron disminuyendo conforme avanzaba el curso, lo que significa que a los alumnos se les dificulta trabajar autónomamente.

Los resultados que se obtuvieron permitieron ver que esta herramienta mostró a los estudiantes conocer las bondades de la plataforma y de esta manera construir su opinión de ella.

El concentrado de actividades y tareas mostró que hubo un faltante de entregas significativas, por lo que al contrastar con las opiniones del cuestionario donde dicen que les pareció muy útil la herramienta no es confiable del todo por el gran número de tareas y actividades no entregadas como lo muestran la imagen 3 y 4 .

\section{CONCLUSIONES}

En esta investigación se conoció la opinión de los estudiantes de licenciatura al usar la plataforma Schoology para realizar las actividades correspondientes a la materia Formación Humana y Social. Como se pudo comprobar el uso de tecnología emergente como la plataforma Schoology, permitió que aún con la pandemia el curso continuara y los alumnos pudieran concluir el curso, aunque sobresale el número alto de participantes que no entregaron las actividades y tareas que se les asignaron, lo que muestra la falta de interés o responsabilidad por la realización de las mismas.

Los temas de ciudadanía así como uso de tecnología son fundamentales para cualquier profesionista, sin embargo se observa por la falta de entrega de tareas y actividades que a los alumnos no les motivó del todo las actividades aunque en sus opiniones ellos argumenten qué si, lo que nos lleva a considerar que aun cuando ya son mayores de edad aún deben tener un acompañamiento y rigor en sus entregas lo que permite observar que no alcanzan una autonomía y responsabilidad en lo que corresponde a sus obligaciones que contraen.

Si en un futuro ellos serán maestros no se aprecia un interés por desarrollar competencias ciudadanas o tecnologías enfocadas a la educación probablemente porque se encuentran en los primeros semestres de la carrera.

La investigación nos da la pauta para realizar otras investigaciones para entender mejor el fenómeno que se observó en esta investigación, por ejemplo, analizar detalladamente los materiales que se propusieron, pudiendo ser que la falta de interés para realizarlos es la descontextualización.

Conocer la opinión del estudiante respecto al cambio repentino de ser un curso presencial al cambio cien por ciento en línea y el enfrentar una situación de tipo emocional al estar ante un problema de salud global como es el covid.19. 


\section{REFERENCIAS BIBLIOGRÁFICAS}

Alcántara, S.A. (enero-junio 2017). Educación cívica y educación ciudadana en México: una perspectiva global y comparada. Revista Española de Educación Comparada, (29), 220-239.

BUAP Benemérita Universidad Autónoma de Puebla. (2016) Programa de la materia Formación Humana y Social. BUAP.

Caballero, A. R. (Septiemre 2016). Educación cívica y formación ciudadana a la luz de la reforma electoral 2014. ¿Qué ciudadanos para qué democracia? Revista del Tribunal Electoral del Poder Judicial de la Federación. Recuperado de https://www.te.gob.mx/eje/media/pdf/ d09b88997d697df.pdf

Concari, S. (2014). Tecnologías emergentes ¿Cuáles usamos?, Latín American Journal of Physics Education, 8(3), 494503.

García, G. O. y González, H. C. A. (febrero 2014) Competencias ciudadanas: Consideraciones desde el concepto de ciudadanía. Revista Plumilla Educativa. 13(1), 373-395.

INEGI Instituto Nacional de Estadística Geografía e Informática. (15 de mayo de 2019). Estadísticas a propósito del día mundial del internet (17 de mayo) Datos nacionales. Comunicado de prensa Núm. 252/19. Recuperado de https://www.inegi.org.mx/contenidos/ saladeprensa/aproposito/2019/ internet2019_Nal.pdf

OEI Organización de Estados Iberoamericanos para la Educación, la Ciencia y la Cultura. (2015). Ciberespacio y Educación. Recuperado de: https://www.oei.es/ historico/divulgacioncientifica/?Ciberes pacio-y-Educacion
OCDE Organización para la Cooperación y el Desarrollo Económico (2005). La definición y selección de competencias clave. Resumen ejecutivo. DeSeCo, 1-20. Recuperado de https://www. deseco.ch/bfs/deseco/en/index/03/02. parsys.78532. downloadList. 94248. DownloadFile.tmp/2005. dscexecutivesummary.sp.pdf

Pérez, Z. R., Mercado, L.P., Martínez, G.M. y Mena, H. E. (enero-junio 2018). La sociedad del conocimiento y la sociedad de la información como la piedra angular en la innovación tecnológica educativa. Revista Iberoamericana para la Investigación y el Desarrollo Educativo, 8(16) $847-870$.

Reyes, M. R. (2012). Creencias pedagógicas y tecnología educativa. México: Bonilla Artigas

SEP Secretaría de Educación Pública. (2007). Programa sectorial de educación 20072012. México: Secretaría de Educación Pública.

SEP Secretaría de Educación Pública. (2011). ModeloEducativo para el Fortalecimiento de Telesecundarias. Documento base. México, D.F.: Secretaría de Educación Pública.

Valencia, M.T. Serna, C.A., Ochoa, A.S., Caicedo T. A., Montes, G.J. y Chávez V. J. (2016). Competencias estándares TIC desde la dimensión pedagógica: Una perspectiva desde los niveles de apropiación de las TIC en la práctica educativa docente. Cali: Pontifica Universidad Javeriana. Cali. 
Zambrano, O. E., Fernández, C. F., Rivera, C.

A. y Zapata, Z. E. (Julio-Diciembre, 2014). Construcción y validación de un instrumento para medir las competencias ciudadanas en estudiantes universitarios. Revista del Instituto de Estudios en Educación Universidad del Norte (21) Recuperado de https://www.redalyc.org/articulo. oa?id=85332835007

\section{ANEXOS:}

Imagen 1. Visualización del curso. Grupo A.

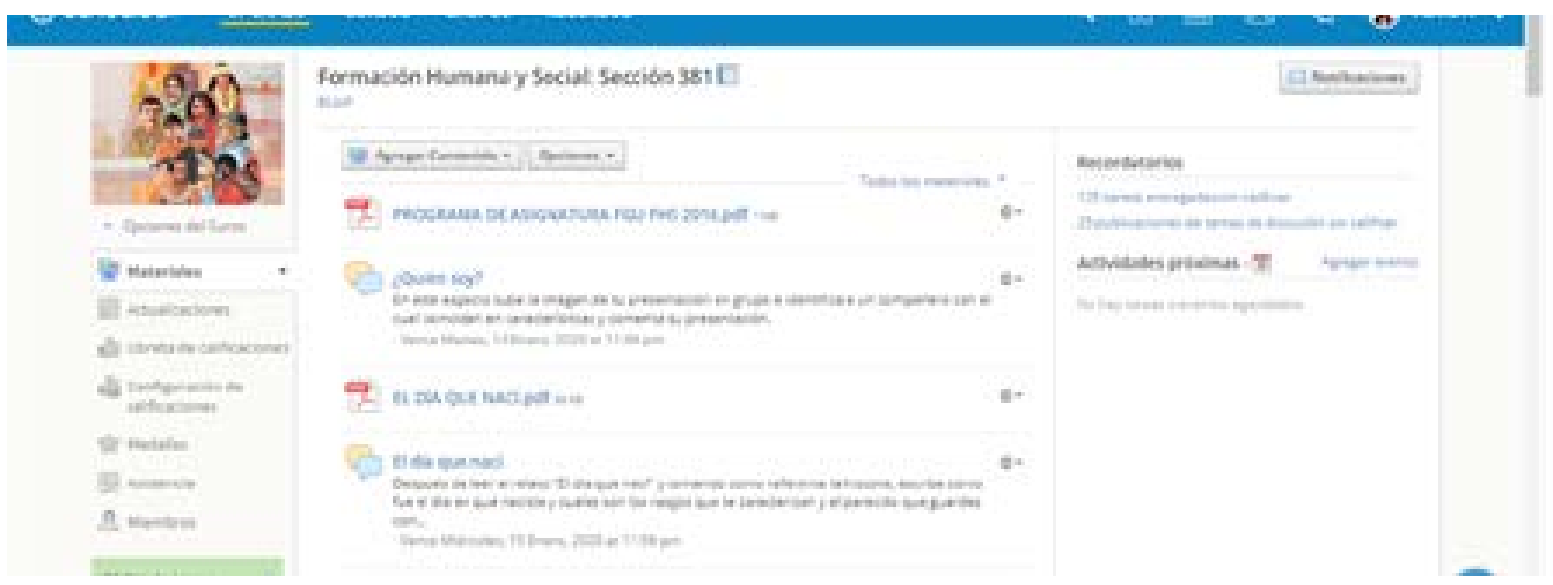

Imagen 2. Visualización del curso. Grupo B.

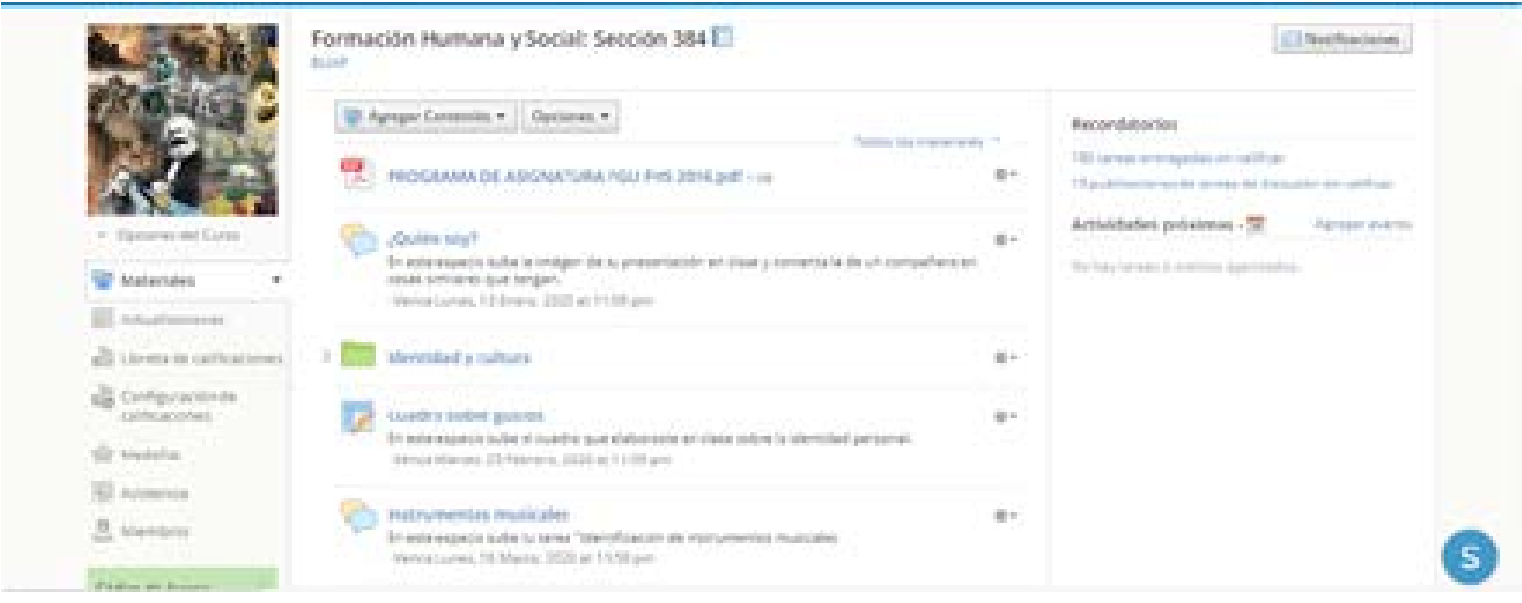



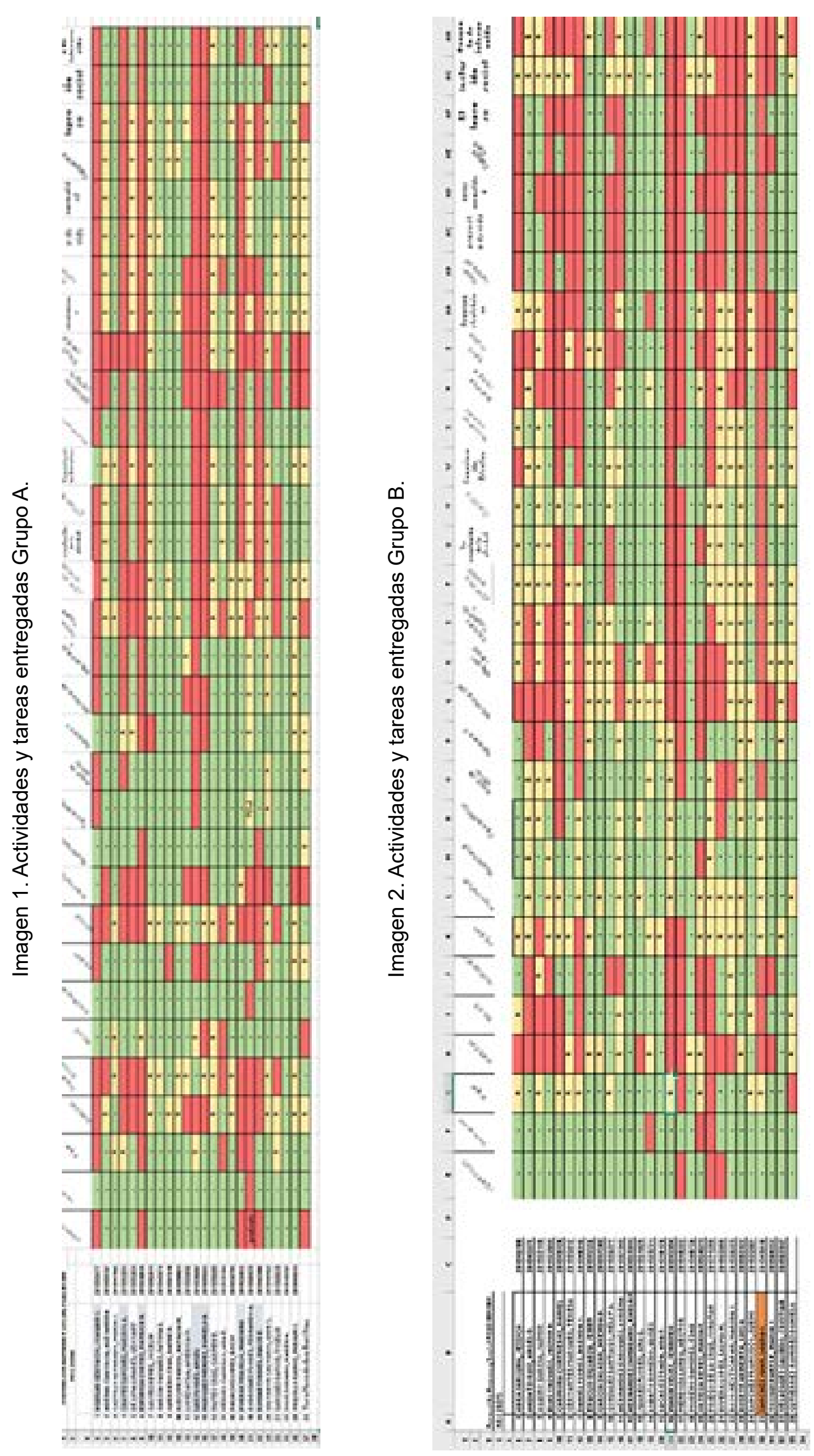